\title{
Incidencia de los accidentes motociclísticos en la mejora de la gestión de prevención de accidentes en la ciudad de Milagro, Ecuador en el año 2017 - 2018
}

\section{(c) (1)(2) (2)}

Incidence of motorcycle accidents in the improvement of accident prevention management in the city of Milagro, Ecuador in 2017 - 2018

Rafael Santamaría Salazar. ${ }^{1} \&$ Jinsop Gamboa Poveda. ${ }^{2}$

Recibido: 30-05-2019 / Revisado: 22-06-2019 /Aceptado: 30-07-2019/ Publicado: 06-09-2019

\section{Abstract \\ DOI: DOI: https://doi.org/10.33262/cienciadigital.v3i3.3.776}

Milagro a city that is part of the province of Guayas that in recent years has been seen in the accelerated growth of the motorcycle park, which is why it is very important to analyze the incidence of traffic accidents that this type of vehicles is causing and that directly affect society The object of study of the investigation and realization of this article emphasizes in reference to the aforementioned based on the research carried out to improve the management in the prevention of these accidents with the sole purpose of doing Awareness and precautionary of the life of the citizens of this sector and that this serves as a model to be executed or to make agreements or strategic alliances with other institutions, whether public or private, of the state and the community, is at the heart of the dynamics of labor performance the experts of the Office of Traffic Accident Investigation (OIAT) of the Transit Commission or of Ecuador in this city, appreciating this reality shows the interrelation between job satisfaction and the performance of the activities of the experts. In virtue of offering an attention aimed at preventing non-conformities from occurring or aggravating the

\footnotetext{
1 Comisión de Transito del Ecuador, rsantamaria@cte.gob.ec

2 Universidad de Milagro,jgamboap@unemi.edu.ec
} 
work of OIAT members, it is intended to create strategies aimed at improving intrinsic - extrinsic factors that affect the satisfaction of these public servants and achieve enhance their individual and social development in them. For which I will be based on the various studies raised in science and provide a small contribution to the scientific community detailing consequences of the phenomenon outlined above and thus improve the prevention of these traffic accidents.

Keywords: Accidents, prevention, management.

\section{Resumen}

Milagro una ciudad que forma parte de la provincia del Guayas que en los últimos años se ha visto en el crecimiento acelerado del parque motociclístico, motivo por el cual es muy importante el análisis de la incidencia de los accidentes de tránsito que este tipo de vehículos está ocasionando y que afectan directamente a la sociedad El objeto de estudio de la investigación y realización del presente artículo hace énfasis en referencia a lo antes mencionado en base a la investigación realizada para mejorar la gestión en la prevención de estos accidentes con el único fin de hacer conciencia y precautelar la vida de los ciudadanos de este sector y que esto sirva de modelo a ejecutarse o a realizar convenios o alianzas estratégicas con las demás instituciones sean públicas o privadas del estado y la comunidad, está en la esencia de la dinámica del desempeño laboral de los peritos de la Oficina de Investigación de Accidentes de Tránsito (OIAT) de la Comisión de Tránsito del Ecuador en esta ciudad, al apreciar esta realidad se evidencia la interrelación entre la satisfacción laboral y el desempeño de las actividades propias de los peritos. En virtud de ofrecer una atención encaminada a prevenir que no se presenten o agraven inconformidades en el trabajo de los miembros de la OIAT, se pretende crear estrategias tendientes a mejorar los factores intrínsecos - extrínsecos, que inciden en la satisfacción de estos servidores públicos y lograr potenciar en ellos su desarrollo individual y social. Para lo cual me fundamentaré en los diversos estudios planteados en la ciencia y brindar un pequeño 
aporte a la comunidad científica detallando consecuencias del fenómeno antes planteado y de esta forma lograr mejorar la prevención de estos accidentes de tránsito. Palabras claves: Accidentes, prevención, gestión.

\section{Introducción}

Las motocicletas se han convertido en un vehículo de preferencia en muchas ciudades del mundo (Nishitateno \& Burke, 2014). La accesibilidad para adquirir este tipo de vehículo se hace factible debido a su costo, facilidad en el desplazamiento, aparcamiento y se explican como un conjunto de factores subjetivos y objetivos que cubren necesidades únicas, que no son iguales a otros tipos de transportación (Marquet \& Miralles-Guasch, 2016).

Alrededor de1,25 millones de personas fallecen anualmente a causa de los accidentes de tránsito (OMS, 2015), entre los usuarios vulnerables de las vías públicas se encuentran los motociclistas, el 23\% de las muertes que ocurren por accidentes de tránsito a nivel mundial involucra este tipo de transporte (Organización Mundial de la Salud, 2013). Del mismo modo alrededor de 50 millones de personas cada año resultan con lesiones no mortales (Salve VIDAS, n.d.), que en algunos casos pueden generar discapacidad y situaciones económicas graves.

Las conductas que asumen los motociclistas en su desplazamiento por las vías son variables (Dapilah, Guba, \& Owusu-Sekyere, 2017), los factores de comportamiento de los conductores (Chu, Wu, Atombo, Zhang, \& Özkan, 2019), pueden ocasionar que infrinjan la ley de tránsito (LOTTTSV, 2014). Existe una normativa que sanciona las contravenciones y delitos de tránsito (Código Integral Penal, 2014), pero podría ser necesario revisar la legislación que rige esa materia ("Fortalecimiento De La Legislación Sobre Seguridad Vial :," n.d.),debido al incremento de los vehículos, tipo motocicletas como medio de transporte en específicamente en esta ciudad del Ecuador en los últimos años.

El consumo de sustancias sujetas a fiscalización y el alcohol no son buenos aliados en la conducción (Carvalho et al., 2016), la velocidad que imprimen los conductores puede provocar siniestros viales (Abdul Manan, Ho, Syed Tajul Arif, Abdul Ghani, \& Várhelyi, 2017)(Eucariotes, 2018), y la no utilización del casco protector no les permite atenuar la 
gravedad de las lesiones (Brijs et al., 2014). Estos se convierten en factores adicionales que desencadenan en algunos accidentes de tránsitos que algunos casos cobran vidas de seres vivos que son importantes en el desarrollo de esta sociedad, la irresponsabilidad por parte de los actores y no cumplimiento con los documentos habilitantes para llevar a cabo la conducción de estos vehículos me ha llevado a la investigación y desarrollo de este artículo. La infraestructura vial y la señalética debe guardar armonía con la circulación de ese tipo de vehículos (Kaygisiz, Senbil, \& Yildiz, 2017), en referencia a lo mencionado por este autor es muy importante generar acciones de cambio o políticas internas a fin de poder controlar la velocidad y la forma de transitar por esta ciudad con el único objetivo de precautelar la vida de los ciudadanos, las condiciones mecánicas de los vehículos influyen en cierta medida en la producción de accidentes de tránsito. Sin embargo en todo momento el conductor es el responsable de su seguridad, la de los pasajeros y la de los demás usuarios viales (Reglamento a LOTTTSV, 2016) aun sabiendo que la ley lo estipula es muy importante el hacer conciencia sobre la forma de actuar y conducir este tipo de vehículos con el único fin de salvar vidas.

La carta magna de la República del Ecuador, establece que la competencia del tránsito le corresponde a los GAD’S Municipales (Asamblea Nacional Constituyente, 2008), por lo que es importante, que la administración pública del Gobierno Autónomo Descentralizado de la ciudad de San Francisco Milagro, ejecute acciones encaminadas a mitigar los accidentes de tránsito en coordinación con el órgano rector y las entidades de control.

La presente investigación tiene como objetivo determinar los principales factores que influyen en la producción de accidentes de motociclistas en la ciudad de San Francisco Milagro, con la finalidad de reducir el número de siniestros viales y de personas que resultan lesionadas o fallecidas.

Apunta a una concientización dirigida a los usuarios de motocicletas a través de programas de educación vial, campañas publicitarias de respeto a las normas tránsito, mejora del control por parte de los órganos competentes. Además de promover la creación de políticas públicas por parte de la administración municipal. 


\section{Revisión de la literatura.}

Los motociclistas continuamente se encuentran expuestos a accidentes de tránsito, relacionándose de forma general a tres factores: humano, vehículo y vía. Sin embargo, el ser humano se ha convertido en un factor de riesgo.

Cuando decimos que el ser humano es un factor riesgo, nos enfocamos en él como conductor, pasajero y peatón. Siendo desde el rol de la conducción, la consideración de los diversos aspectos que influyen en su comportamiento.

Los motociclistas deben contar con la total capacidad para conducir un vehículo motorizado de dos ruedas. Según los resultados de los "análisis de accidentes constituyen una de las principales preocupaciones en materia de seguridad vial. A pesar de la importancia del factor humano en los accidentes de motocicleta, la investigación académica aún no satisface la necesidad de una mejor comprensión de la actividad de la conducción. Se han llevado a cabo discusiones de grupos focales con corredores para obtener información sobre la naturaleza de la conducción, los factores de riesgo que subyacen en esta actividad, así como cuestiones estratégicas y tácticas"(Huth, Füssl, \& Risser, 2014).

Al referirnos a accidentes de tránsito, nos adentramos a un tema neurálgico de la sociedad, puesto que es una de las principales causas de muerte a nivel mundial, por ende, en el Ecuador. El tratamiento de esta problemática es de tal importancia, que instituciones a nivel mundial han hecho pronunciamientos. Estos esfuerzos no garantizan la efectividad de la gestión pública y el impacto generado aún no es suficiente.

Según datos de la INEC, 2018 en el Ecuador fallecen al menos 6 personas diarias por accidentes de tránsito, y existe cerca de 55 personas que resultan lesionados por estos sucesos, siendo cifra alarmante que deja claro un déficit en cuanto a programas de prevención y seguridad vial que ayuden a disminuir la tasa de mortalidad y morbilidad causada por los accidentes que existen en el país, en estos accidentes no solo son las vidas las que se pierden, que son la parte fundamental del problema, porque tras de una muerte desencadena un sin número de situaciones sociales, sino que también deja pérdidas económicas al Estado (Román, 2015). 
Las superficies de las calzadas, así como el uso de los suelos mixtos por parte de los motociclistas aumentan el riesgo de los accidentes, por lo cual es importante el uso de medidas proactivas. A medida que las áreas urbanas de los países en desarrollo están cambiando rápidamente junto con el aumento de la motorización, la definición de los factores que contribuyen de accidentes hará que sea posible tomar medidas proactivas mediante la planificación de las políticas de diseño urbano, en lugar de esperar a que los accidentes a pasar a tomar medidas reactivas (Kaygisiz et al., 2017).

La presente investigación, servirá de aporte para brindar a la sociedad varias alternativas de solución, al gran impacto que producen los accidentes de tránsito. Pretende integrar a los usuarios viales, sociedad y Estado con la finalidad de reducir la siniestralidad de tránsito.

\section{Metodología.}

El diseño utilizado en la presente investigación de tipo documental, es realizada por estudio de casos, con base en el reporte de partes policiales por accidentes de la Comisión de Tránsito del Ecuador, ente de control en la circunscripción territorial del GAD Municipal.

Los datos observados, corresponden a las estadísticas de accidentes en los años 2017 y 2018, de la Agencia Nacional de Tránsito y la Comisión de Tránsito del Ecuador (https://www.comisiontransito.gob.ec/biblioteca/\#), en donde interviene al menos un motociclista en los accidentes de tránsito.

El estudio se realizó en la ciudad de San Francisco de Milagro, la misma que se encuentra ubicada al centro-sur de la región Litoral del Ecuador, Provincia del Guayas de las cuales es una de las ciudades más pobladas. La mayoría de las vías son lastradas, pero se puede apreciar que se encuentran en un proceso de regeneración urbana.

El objetivo pretende determinar los factores que inciden en la ocurrencia de los accidentes (enfoque multifactorial), considera el número de accidentes de tránsito en donde intervienen motociclistas (enfoque cualitativo).

Se realiza una comparación de los datos obtenidos en las estadísticas mostradas por la ANT (https://www.ant.gob.ec/index.php/noticias/estadisticas), Agencia Nacional de Tránsito para los años 2017 y 2018, se medirán indicadores como el número personas fallecidas por el total de accidentes de tránsito, número de personas lesionadas por el total de accidentes de tránsito, 
número de vehículos tipo motocicleta por el total de los vehículos involucrados en los siniestros de tránsito.

\section{Presentación y análisis de resultados.}

El análisis se efectúa entre el número de accidentes de tránsito entre el 2017 y 2018 a nivel nacional, por provincias y cantones; la mortalidad va en relación al número de personas fallecidas y el número total de accidentes de tránsito; la morbilidad como la relación entre el número de personas lesionadas respecto al total de siniestros de tráfico. Así como la proporción de vehículos tipo motocicleta involucradas en los siniestros de tránsito en la ciudad de Milagro.

\section{Los accidentes de tránsito a nivel nacional}

Las muertes violentas por accidentes de tránsito ocupan el sexto lugar de las causas de mortalidad en la República del Ecuador (INEC, 2018), a continuación, detallamos el total de accidentes de tránsito ocurridos en el Ecuador en los años 2017 - 2018, así como el total de personas fallecidas y lesionadas a causa de esos siniestros (tabla 1).

Tabla 1. Accidentes de transito en el Ecuador y consecuencias.

\begin{tabular}{cccc}
\hline \multirow{2}{*}{ AÑO } & SINIESTROS & $\begin{array}{c}\text { PERSONAS } \\
\text { FALLECIDAS }\end{array}$ & $\begin{array}{c}\text { PERSONAS } \\
\text { LESIONADAS }\end{array}$ \\
\hline 2017 & $28.967,00$ & $2.153,00$ & $22.018,00$ \\
2018 & $25.530,00$ & $2.151,00$ & $19.858,00$ \\
\hline
\end{tabular}

Tabla 2. Siniestros de tránsito, personas fallecidas y lesionadas por provincias

\begin{tabular}{lcccccc}
\hline PROVINCIAS & $\begin{array}{c}\text { SINIESTROS } \\
\mathbf{2 0 1 7}\end{array}$ & $\begin{array}{c}\text { SINIESTROS } \\
\mathbf{2 0 1 8}\end{array}$ & $\begin{array}{c}\text { PERSONAS } \\
\text { FALLECIDAS } \\
\mathbf{2 0 1 7}\end{array}$ & $\begin{array}{c}\text { PERSONAS } \\
\text { FALLCIDAS } \\
\mathbf{2 0 1 8}\end{array}$ & $\begin{array}{c}\text { PERSONAS } \\
\text { LESIONADAS } \\
\mathbf{2 0 1 7}\end{array}$ & $\begin{array}{c}\text { PERSONAS } \\
\text { LESIONADAS } \\
\mathbf{2 0 1 8}\end{array}$ \\
\hline Azuay & 1497 & 1528 & 92 & 102 & 1127 & 1357 \\
Guayas & 8422 & 8619 & 456 & 511 & 8081 & 7819 \\
Imbabura & 1324 & 358 & 81 & 45 & 489 & 295 \\
Los Ríos & 903 & 837 & 180 & 197 & 949 & 927 \\
Manabí & 1305 & 1173 & 150 & 141 & 1141 & 935 \\
Pichincha & 9361 & 7599 & 375 & 357 & 5343 & 4521 \\
Tungurahua & 1120 & 1407 & 92 & 73 & 761 & 689 \\
$\begin{array}{l}\text { Resto de provincias } \\
\text { del país (17) }\end{array}$ & 5035 & 4009 & 727 & 725 & 4127 & 3315 \\
\hline
\end{tabular}




\section{Los accidentes de tránsito a nivel provincial.}

El Ecuador tiene 24 provincias, por lo que sería importante desagregar los datos nacionales totales por provincias, con la finalidad de adentrarnos a nuestro campo de estudio (tabla 2). Analizando los datos desagregados del total nacional, tenemos que Guayas y Pichincha, son las provincias en donde se generan la mayor cantidad de accidentes de tránsito, así como el mayor número de personas que resultan lesionadas; En el estudio se identifica a estas dos provincias en la demanda de mayor atención por parte de los organismos competentes en materia de tránsito, en la producción de políticas públicas, ordenanzas, controles entre otros, a fin de disminuir la producción de siniestros viales.

\section{Los accidentes de tránsito por cantones de la provincia del Guayas.}

La ciudad donde efectuamos nuestro estudio, pertenece a la provincia del Guayas, por tal razón es importante conocer el número de accidentes de tránsito que se suscitan en los diferentes cantones que conforman la circunscripción territorial de la Provincia del Guayas. Del mismo modo el total de personas lesionadas y fallecidas (tabla 3).

Tabla 3. Siniestros de tránsito, personas lesionadas y fallecidas en los cantones de Guayas.

\begin{tabular}{|c|c|c|c|c|c|c|}
\hline CANTONES & $\begin{array}{c}\text { SINIESTROS } \\
2017\end{array}$ & $\begin{array}{l}\text { SINIESTROS } \\
2018\end{array}$ & $\begin{array}{c}\text { PERSONAS } \\
\text { FALLECIDAS } \\
2017\end{array}$ & $\begin{array}{c}\text { PERSONAS } \\
\text { FALLECIDAS } \\
2018\end{array}$ & $\begin{array}{c}\text { PERSONAS } \\
\text { LESIONADAS } \\
2017\end{array}$ & $\begin{array}{c}\text { PERSONAS } \\
\text { LESIONADAS } \\
2018\end{array}$ \\
\hline *Guayaquil & 5044 & 5098 & 159 & 184 & 4667 & 4276 \\
\hline Daule & 336 & 392 & 21 & 33 & 340 & 391 \\
\hline Durán & 565 & 512 & 26 & 23 & 546 & 443 \\
\hline Milagro & 570 & 632 & 25 & 14 & 574 & 650 \\
\hline Naranjal & 279 & 300 & 30 & 42 & 276 & 315 \\
\hline Samborondón & 233 & 236 & 12 & 11 & 199 & 186 \\
\hline Yaguachi & 229 & 222 & 55 & 25 & 256 & 251 \\
\hline $\begin{array}{l}\text { Resto de cantones } \\
\text { de la provincia (19) }\end{array}$ & 1168 & 1227 & 128 & 179 & 1223 & 1307 \\
\hline
\end{tabular}

* La ciudad de Guayaquil es el único cantón que cuenta con agentes civiles de tránsito en la provincia del Guayas, en ella no mantiene el control de tránsito el organismo del ejecutivo, la Comisión de tránsito del Ecuador, además su extensión territorial y poblacional es superior al resto de cantones de la provincia por la cual la vamos a exceptuar de este análisis.

La ciudad de San Francisco de Milagro es el cantón donde se generan la mayor cantidad de accidentes de tránsito y personas lesionadas a consecuencia de los siniestros, exceptuando a la ciudad de Guayaquil. 
Para el año 2017 se encontraron inmersos en accidentes de tránsito dentro de la provincia del Guayas un total de 5924 vehículos (tabla 4).

Tabla 4. Tipo de vehículos involucrados en siniestros de tránsito provincia del Guayas 2017.

\begin{tabular}{lc}
\hline \multicolumn{1}{c}{ TIPO DE VEHICULO } & SINIESTRO \\
\hline AUTOMOVIL & 1.118 \\
MOTOCICLETA & 1229 \\
CAMIONETA & 619 \\
UTILITARIO & 320 \\
BUS & 264 \\
CAMIÓN & 459 \\
OTROS* & 124 \\
TRICIMOTO & 26 \\
BICICLETA - TRICICLO & 63 \\
NO IDENTIFICADO** & 1702 \\
TOTAL & $\mathbf{5 . 9 2 4}$ \\
\hline
\end{tabular}

*Otros (tráileres, volquetas, tanqueros, grúas, especiales, agrícolas, otros)

**No identificado (abandonaron el lugar no se conoce las características)

Mientras que para el 2018 se redujo a 5854 el número de vehículos involucrados en siniestros de tránsito (tabla 5). Se excluye el número de vehículos accidentados en la ciudad de Guayaquil para ambos períodos.

Tabla 5. Tipo de vehículos involucrados en siniestros de tránsito provincia del Guayas 2018.

\begin{tabular}{lc}
\hline \multicolumn{1}{c}{ TIPO DE VEHICULO } & SINIESTRO \\
\hline AUTOMOVIL & 960 \\
MOTOCICLETA & 1193 \\
CAMIONETA & 548 \\
UTILITARIO & 296 \\
BUS & 204 \\
CAMIÓN & 382 \\
OTROS* & 152 \\
TRICIMOTO & 21 \\
BICICLETA - TRICICLO & 59 \\
NO IDENTIFICADO** & 2039 \\
TOTAL & $\mathbf{5 . 8 5 4}$ \\
\hline
\end{tabular}

*Otros (tráileres, volquetas, tanqueros, grúas, especiales, agrícolas, otros)

**No identificado (abandonaron el lugar no se conoce las características)

Se puede apreciar que el tipo de vehículo que más se encuentra inmerso en los siniestros de tránsito para ambos períodos, son las motocicletas.

\section{Los accidentes de tránsito en la ciudad de San Francisco de Milagro-Ecuador.}

En este párrafo detallaremos el número de accidentes de tránsito que involucran motocicletas, las personas que resultaron lesionadas y fallecidas a causa de estos, en la ciudad de San 
Francisco de Milagro para los años 2017, 2018 y los suscitados hasta el 05 de julio del 2019 (tabla 6).

Tabla 6. Accidentes que involucran motocicletas, lesionados y fallecidos.

\begin{tabular}{|c|c|c|c|c|c|c|c|c|c|}
\hline \multirow{3}{*}{$\begin{array}{l}\text { Cantón } \\
\text { Milagr }\end{array}$} & \multicolumn{3}{|c|}{ TOTAL DE SINIESTROS } & \multicolumn{3}{|c|}{ TOTAL DE FALLECIDOS } & \multicolumn{3}{|c|}{ TOTAL DE LESIONADOS } \\
\hline & 2017 & 2018 & 2019 & 2017 & 2018 & 2019 & 2017 & 2018 & 2019 \\
\hline & 156 & 139 & 137 & 8 & 5 & 9 & 158 & 113 & 219 \\
\hline
\end{tabular}

Del mismo modo se cuantificará el total de vehículos de tipo motocicleta que hayan participado en los siniestros de tránsito, dentro de la circunscripción territorial del cantón Milagro (tabla 7).

Se analizará cuáles son los determinantes en la producción de accidentes de tránsito de motociclistas, es decir, las causas por las que se originan estos siniestros (inobservancia, imprudencia, impericia, ingesta alcohólica, configuración vial). Con el estudio realizado, se observa que después de Guayaquil, San Francisco de Milagro es la segunda ciudad donde se producen la mayor cantidad de siniestros de tránsito y personas lesionadas en la Provincia del Guayas (tabla 8).

Tabla 7. Vehículos involucrados en los siniestros de tránsito en el cantón Milagro para los años 2017, 2018 hasta inicios del tercer trimestre del 2019.

\begin{tabular}{lccc}
\hline \multicolumn{1}{c}{ VEHICULO } & 2017 & 2018 & 2019 \\
\hline AUTOMÓVIL & 150 & 122 & 49 \\
BICICLETA & 17 & 21 & 23 \\
CAMIÓN & 28 & 26 & 15 \\
CAMIONETA & 69 & 78 & 41 \\
ESPECIAL & 1 & 2 & - \\
JEEP & 19 & 27 & 16 \\
MOTOCICLETA & 243 & 308 & 184 \\
NO IDENTIFICADO & 330 & 390 & 199 \\
OMNIBUS & 14 & 20 & 15 \\
TRAILER & - & 2 & 2 \\
TRICICLO & 4 & 1 & 3 \\
VOLQUETA & - & 1 & - \\
TOTALES & 875 & 998 & 351 \\
\hline
\end{tabular}


ISSN: 2602-8085

Tabla 8. Causa probable de los accidentes con motocicletas para los años 2017, 2018 hasta inicios del tercer trimestre del 2019.

\begin{tabular}{|c|c|c|c|c|}
\hline $\begin{array}{l}\text { CAUSA PROBABLE DE LOS ACCIDENTES } \\
\end{array}$ & SINIESTROS & 2017 & 2018 & 2019 \\
\hline REALIZAR CAMBIO BRUSCO O INDEBIDO DE CARRIL & 34 & 24 & 3 & 7 \\
\hline CONDUCIR DESATENTO A LAS CONDICIONES DEL TRÁNSITO & 143 & 65 & 55 & 23 \\
\hline $\begin{array}{l}\text { CONDUCE BAJO INFLUENCIA DE ALCOHOL, SUSTANCIAS } \\
\text { ESTUPEFACIENTES O PSICOTROPICAS Y/O MEDICAMENTOS }\end{array}$ & 1 & 1 & - & - \\
\hline $\begin{array}{l}\text { NO TRANSITAR POR LAS ACERAS O ZONAS DE SEGURIDAD } \\
\text { DESTINADAS PARA EL EFECTO }\end{array}$ & 1 & 1 & - & - \\
\hline $\begin{array}{l}\text { NO RESPETAR LAS SEÑALES REGLAMENTARIAS DE (PARE, CEDA EL } \\
\text { PASO, LUZ ROJA DEL SEMAFORO, ETC) }\end{array}$ & 48 & 21 & 21 & 6 \\
\hline $\begin{array}{l}\text { NO MANTENER LA DISTANCIA PRUDENCIAL CON RESPECTO AL } \\
\text { VEHICULO QUE LE ANTECEDE }\end{array}$ & 40 & 10 & 14 & 16 \\
\hline $\begin{array}{l}\text { NO GUARDAR LA DISTANCIA LATERAL MINIMA DE SEGURIDAD ENTRE } \\
\text { VEHICULOS }\end{array}$ & 88 & 3 & 31 & 54 \\
\hline $\begin{array}{l}\text { BAJARSE O SUBIRSE DE VEHICULOS EN MOVIMIENTO SIN TOMAR LAS } \\
\text { PRECAUCIONES DEBIDAS }\end{array}$ & 30 & 10 & & 20 \\
\hline $\begin{array}{l}\text { NO CEDER EL DERECHO DE VIA O PREFERENCIA DE PASO A } \\
\text { VEHICULOS Y/O PEATON }\end{array}$ & 33 & 11 & 11 & 11 \\
\hline $\begin{array}{l}\text { CASO FORTUITO O FUERZA MAYOR (EXPLOSIÓN DE NEUMATICO } \\
\text { NUEVO, DERRUMBE, INUNDACIÓN, CAIDA DE PUENTE, ARBOL, } \\
\text { PRESENCIA INTEMPESTIVA E IMPREVISTA DE SEMOVIENTES EN LA } \\
\text { VIA. ETC) }\end{array}$ & 2 & 2 & - & - \\
\hline $\begin{array}{l}\text { CONDUCIR EN SENTIDO CONTRARIO A LA VIA NORMAL DE } \\
\text { CIRCULACIÓN }\end{array}$ & 7 & 5 & 2 & - \\
\hline CONDUCIR VEHICULO SUPERANDO LOS LIMITES DE VELOCIDAD & 1 & 1 & & - \\
\hline DEJAR O RECOGER PASAJEROS EN LUGARES NO PERMITIDOS & 4 & 2 & 2 & - \\
\hline TOTALES & 432 & 156 & 139 & 137 \\
\hline
\end{tabular}

Para el año 2017 se produjeron un total de 570 accidentes de tránsito, de los cuales en 156 se encuentran involucradas motocicletas. Mientras que para el 2018 la cifra de accidentes de tránsito se incrementó a 632, de los cuales en 139 estaban involucradas motocicletas. Mientras qua hasta inicio del mes de julio del 2019 ya se han producido 137 accidentes donde involucran motocicletas. Por su parte las personas lesionadas ascendieron de 574 en el 2017 a 650 para el 2018 del total de accidentes, sin embargo, en lo que se refiere a accidentes en motocicletas disminuyó de 158 en el 2017 a 113 para el 2018. Pero hasta inicios del segundo semestre del 2019 esta cifra ya fue superada, alcanzando a 219 la cifra de lesionados productos de siniestros en motocicletas. 
El número de muertes violentas por accidentes de tránsito fue de 25 para el año 2017 y se ha logrado reducir a 14, para el año 2018 en ese cantón, en donde se registró que la muerte por accidentes de motocicletas fue de 8 para el 2017 y se redujo a 5 para el 2018. Sin embargo, hasta inicios del tercer trimestre del año 2019, ya se han registrado 9 muertes por accidentes de tránsito que involucran motocicletas.

Gráfico 1. Siniestros por año y meses.

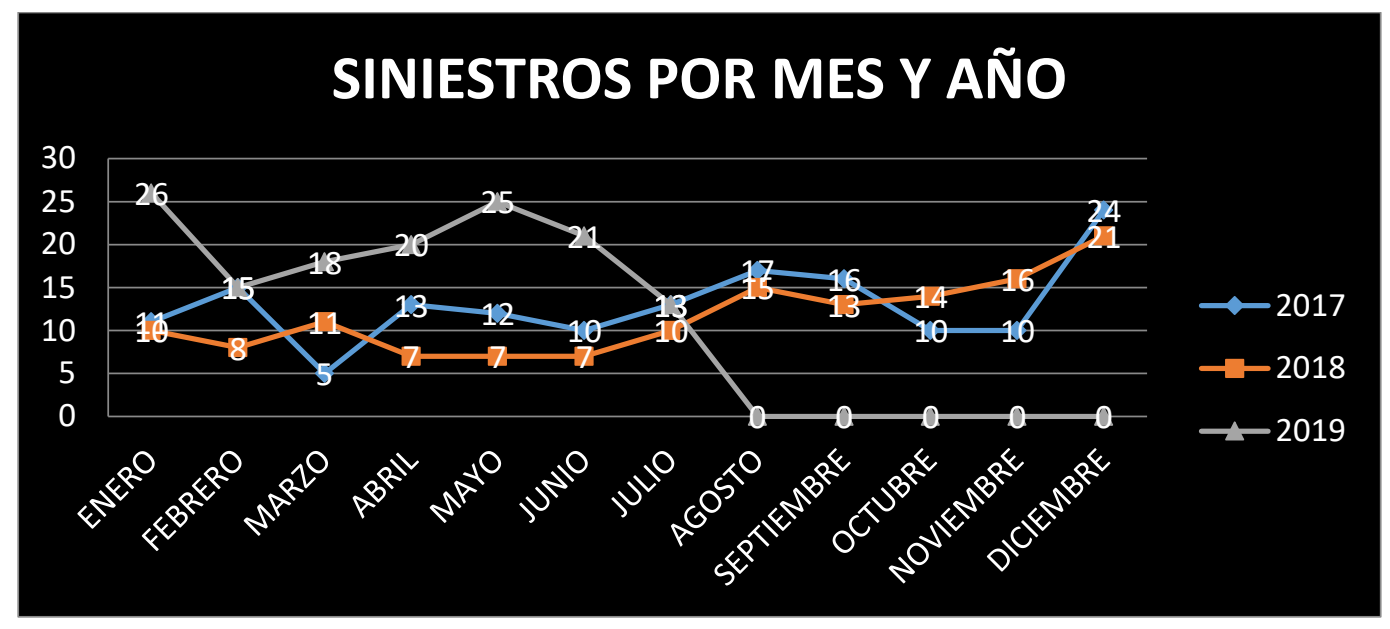

Fuente: Unidad de Estadísticas del Departamento de Planificación de la CTE, sistema AXIS.

Gráfico 2. Fallecidos por año y meses.

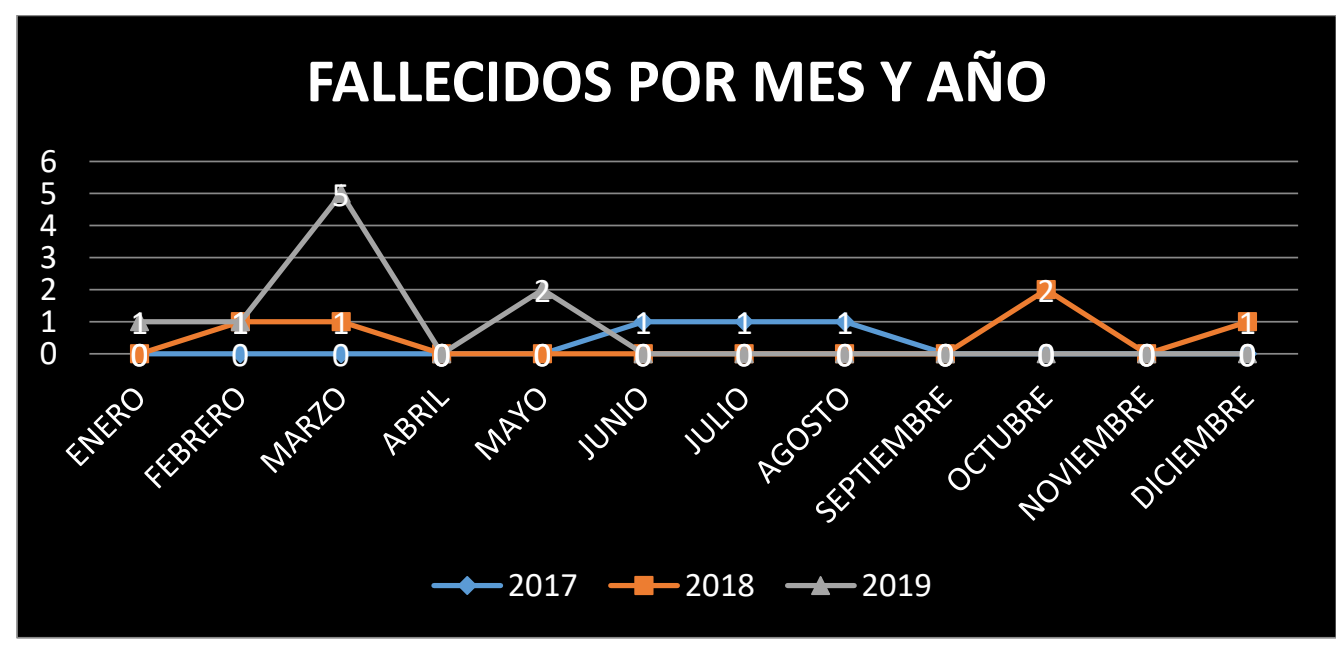

Fuente: Unidad de Estadísticas del Departamento de Planificación de la CTE, sistema AXIS. 
Gráfico 3. Heridos por mes y año.

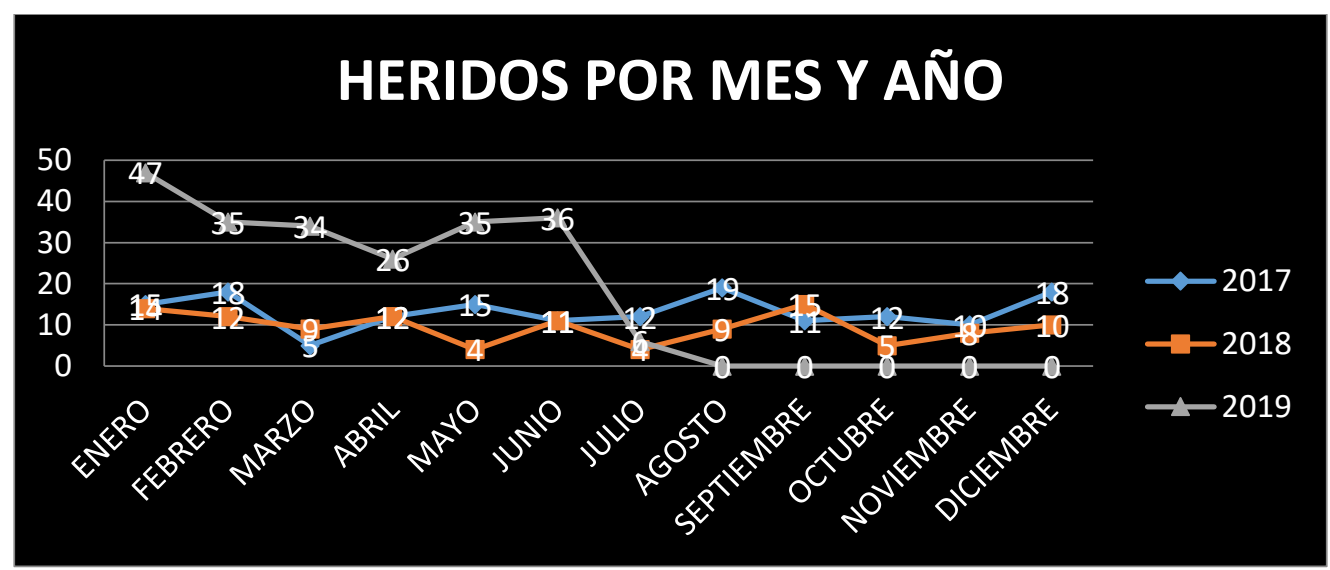

Fuente: Unidad de Estadísticas del Departamento de Planificación de la CTE, sistema AXIS.

Gráfico 4. Siniestros, fallecidos y heridos por año.

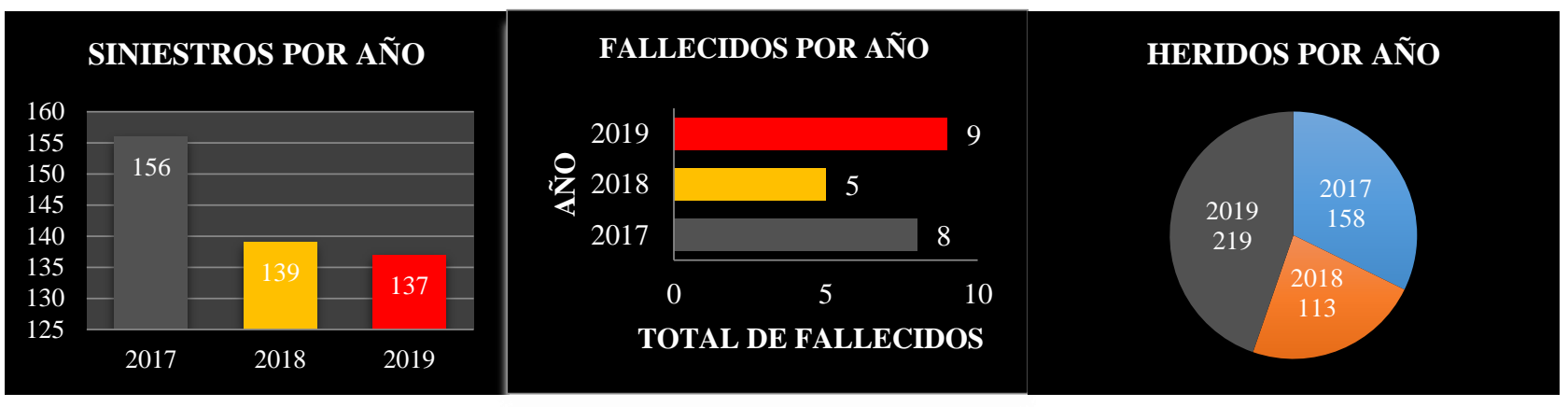

Fuente: Unidad de Estadísticas del Departamento de Planificación de la CTE, sistema AXIS.

\section{Integración de resultados y conclusiones}

Con el presente estudio hemos observado, que la ciudad de San Francisco de Milagro en la República del Ecuador, es la segunda ciudad dentro de la provincia del Guayas con el mayor número de siniestros viales y personas que resultan lesionadas para los años 2017 y 2018, habiendo sufrido un incremento en el último año.

La movilización en los vehículos de tipo motocicleta es más atractivo para los residentes en el cantón Milagro, por cuanto la población opta por adquirir un vehículo de esa categoría, atribuyéndole factores como la accesibilidad para su adquisición y el costo. Es notorio la presencia de motocicletas y como estas se incrementan cada vez más en el cantón Milagro, 
no se ha podido cuantificar el número de motocicletas matriculadas en las dependencias de la EMOVIN (empresa pública municipal de transporte), a pesar de solicitarles la información, estos no colaboran con la información correspondiente del parque vehicular de tipo motocicleta para los años 2017 y 2018, tampoco registran información en un portal web, pese a los avances tecnológicos propios del siglo XXI. Lo que dificulta el estudio realizado, debido a la falta de colaboración por parte de la empresa pública de transporte, perteneciente a la administración pública municipal.

Aunque existen preferencias en los milagreños por el uso de motocicletas, no se puede dejar de lado que estos implican factores de riesgo, como los que ya hemos descrito en este estudio. Además, los traumas que se producen en el cuerpo humano son superiores a los que se generarían en un vehículo de cuatro ruedas.

Adicionalmente la circulación de motocicletas se ha transformado en un problema social en la ciudad, debido a que conductores la utilizan como medio de transporte remunerado de pasajeros, al margen de la ley. Siendo esto objeto de otro estudio.

Dentro de las tres principales determinantes para la ocurrencia de siniestros de tránsito en motocicletas dentro del cantón Milagro, hemos podido encontrar las siguientes: 1. Conducir desatento a las condiciones del tránsito y entorno de la vía; 2 . No conservar la distancia lateral de seguridad mínima entre vehículos; 3 . No respetar las señales de tránsito (pare, ceda el paso, semáforos entre otros); 4 . No conservar la distancia de seguimiento con el vehículo que le antecede; 5 . Realizar cambio brusco o indebido de carril.

Luego de encontrar los determinantes que producen los siniestros de tránsito en la ciudad de San Francisco de Milagro, queremos aportar en la mejora de la gestión de las instituciones que ejercen el control del tránsito, a través de campañas de Educación Vial, concientización en el uso del casco protector, respeto a las normas de tránsito, manejo a la defensiva.

De igual manera a la administración pública del GAD Municipal, lo conminamos a la creación de ordenanzas que protejan a sus ciudadanos en lo que respecta a la seguridad vial, restricción de circulación de más de una persona del mismo sexo en fines de semana y a determinadas horas y lugares, mejora de la infraestructura vial carriles exclusivos para motocicletas. 
Proponer sea analizada por los asambleístas una reforma legal que contemple sanciones más severas a quienes utilicen este tipo de vehículos como medio de transporte remunerado, tales como suspensión temporal de la licencia de conducir y sanciones pecuniarias más elevadas, en caso de reincidencia suspensión definitiva de la licencia de conducir y un incremento de la sanción pecuniaria en doble de su valor.

Regulaciones a los importadores de motocicletas, tales como que únicamente efectúen las ventas de motocicletas a personas que posean licencia de conducir de la categoría requerida para ese tipo de vehículos, obligatoriedad de vender casco homologados, obligatoriedad de haber aprobado un curso de educación vial en una dependencia certificada en materia de tránsito.

En lo que respeta al ente de control de tránsito, reforzar el servicio en el perímetro urbano y rural, para evitar que los conductores infrinjan la ley, a través de operativos rigurosos, presencia de un mayor número de vehículos policiales dentro de sus rondas como medio de disuasión para posibles infractores.

Este estudio tiene como objetivo mejorar la gestión en la prevención y disminuir el número de siniestros de tránsito de motociclistas en el GAD Municipal de San Francisco de Milagro, así como también reducir el número de lesionados y víctimas mortales en motocicletas.

\section{Bibliografía}

Abdul Manan, M. M., Ho, J. S., Syed Tajul Arif, S. T. M., Abdul Ghani, M. R., \& Várhelyi, A. (2017). Factors associated with motorcyclists' speed behaviour on Malaysian roads. Transportation Research Part F: Traffic Psychology and Behaviour, 50(5), 109-127. https://doi.org/10.1016/j.trf.2017.08.006

Brijs, K., Brijs, T., Sann, S., Trinh, T. A., Wets, G., \& Ruiter, R. A. C. (2014). Psychological determinants of motorcycle helmet use among young adults in Cambodia. Transportation Research Part F: Traffic Psychology and Behaviour, 26(PA), 273-290. https://doi.org/10.1016/j.trf.2014.08.002

Carvalho, H. B. de, Andreuccetti, G., Rezende, M. R., Bernini, C., Silva, J. S., Leyton, V., \& 
D’Andréa Greve, J. M. (2016). Alcohol and drug involvement in motorcycle driver injuries in the city of Sao Paulo, Brazil: Analysis of crash culpability and other associated factors. Drug and Alcohol Dependence, 162, 199-205. https://doi.org/10.1016/j.drugalcdep.2016.03.007

Chu, W., Wu, C., Atombo, C., Zhang, H., \& Özkan, T. (2019). Traffic climate, driver behaviour, and accidents involvement in China. Accident Analysis and Prevention, 122(1178), 119-126. https://doi.org/10.1016/j.aap.2018.09.007

Constitutivos, E., \& Estado, D. E. L. (2011). Constitucion de la republica del ecuador 2008, $1-136$.

Constituyente, A. N. (2014). Codigo Orgánico Integral Penal. Noticias, 144. https://doi.org/10.1196/annals.1389.023

Dapilah, F., Guba, B. Y., \& Owusu-Sekyere, E. (2017). Motorcyclist characteristics and traffic behaviour in urban Northern Ghana: Implications for road traffic accidents. Journal of Transport and Health, 4, 237-245. https://doi.org/10.1016/j.jth.2016.03.001

De, R. O. S. (2014). LEY ORGANICA DE TRANSPORTE TERRESTRE TRANSITO Y SEGURIDAD VIAL, 1-66.

Diego Xavier Román Matamoros María Gracia Calisto, MSc., Director de Trabajo de Titulación. (n.d.).

Eucariotes, G. E. N. (2018). Control De La, 31(1), 67-83. https://doi.org/10.4067/S003498872003001000019

Fortalecimiento De La Legislación Sobre Seguridad Vial: (n.d.).

Huth, V., Füssl, E., \& Risser, R. (2014). Motorcycle riders' perceptions, attitudes and strategies: Findings from a focus group study. Transportation Research Part F: Traffic $\begin{array}{lllll}\text { Psychology and } \quad \text { Behaviour, } & \text { 25(PART } & \text { A), 85. }\end{array}$ https://doi.org/10.1016/j.trf.2014.05.004

Kaygisiz, Ö., Senbil, M., \& Yildiz, A. (2017). Influence of urban built environment on traffic 
accidents: The case of Eskisehir (Turkey). Case Studies on Transport Policy, 5(2), 306313. https://doi.org/10.1016/j.cstp.2017.02.002

Marquet, O., \& Miralles-Guasch, C. (2016). City of Motorcycles. On how objective and subjective factors are behind the rise of two-wheeled mobility in Barcelona. Transport Policy, 52, 37-45. https://doi.org/10.1016/j.tranpol.2016.07.002

Nishitateno, S., \& Burke, P. J. (2014). The motorcycle Kuznets curve. Journal of Transport Geography, 36(2014), 116-123. https://doi.org/10.1016/j.jtrangeo.2014.03.008

Organizacion Mundial de la Salud. (2013). La Seguridad Vial 2013. 1211 Ginebra 27, 1, 12.

Salud, O. M. de la. (2015). La Seguridad Vial 2015, 12. Retrieved from www.who. int

Salve VIDAS. (n.d.).

Vial, S. (2016). REGLAMENTO A LEY DE TRANSPORTE TERRESTRE, 1-97.

URL: https://www.comisiontransito.gob.ec/biblioteca/\#

URL: https://www.ant.gob.ec/index.php/noticias/estadisticas

URL: http://www.ecuadorencifras.gob.ec/estadisticas/

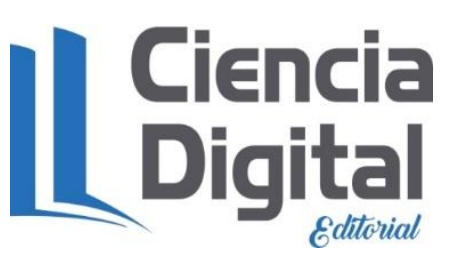




\section{PARA CITAR EL ARTÍCULO INDEXADO.}

Santamaría Salazar, R., \& Gamboa Poveda, J. (2019). Incidencia de los accidentes motociclísticos en la mejora de la gestión de prevención de accidentes en la ciudad de Milagro, Ecuador en el año 2017 - 2018. Ciencia Digital, 3(3.3), 144-161. https://doi.org/10.33262/cienciadigital.v3i3.3.776

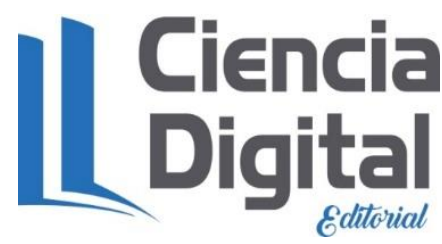

El artículo que se publica es de exclusiva responsabilidad de los autores y no necesariamente reflejan el pensamiento de la Revista Ciencia Digital.

El artículo queda en propiedad de la revista y, por tanto, su publicación parcial y/o total en otro medio tiene que ser autorizado por el director de la Revista Ciencia Digital.
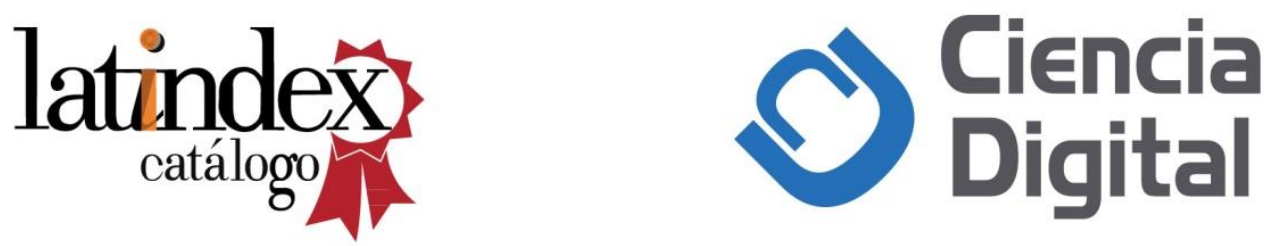